\title{
Erratum to: Acoustic radiation force on an air bubble and soft fluid spheres in ideal liquids: Example of a high-order Bessel beam of quasi-standing waves
}

\section{F.G. Mitri ${ }^{\mathrm{a}}$}

Los Alamos National Laboratory, MPA-11, Sensors \& Electrochemical Devices, Acoustics \& Sensors Technology Team, MS D429, Los Alamos, NM 87545, USA

Original article: Eur. Phys. J. E (2009) 28: 469, DOI: 10.1140/epje/i2009-10449-y

Received 27 October 2012 and Received in final form 15 November 2012

Published online: 21 December 2012 - (c) EDP Sciences / Società Italiana di Fisica / Springer-Verlag 2012

In the paper in question [1], errors of transcriptions have occurred and some equations and results are corrected herein. In particular, the factor $(-1)^{m}$ that multiplies the reflected wave's amplitude $\Phi_{1}$ was missed. In the following, the corrected equations are provided along with related computational results.

Regarding eq. (2), the incident velocity potential should have been written as

$$
\Phi_{J_{m}, q s t}^{(\mathrm{inc})}=e^{-i \omega t} \sum_{n=|m|}^{\infty} \frac{(n-m) !}{(n+m) !}(2 n+1) i^{(n-m)} j_{n}(k r)\left[\Phi_{0} e^{i k_{z} h}+\Phi_{1}(-1)^{n+m} e^{-i k_{z} h}\right] P_{n}^{m}(\cos \theta) P_{n}^{m}(\cos \beta) e^{i m \phi} .
$$

It follows that the scattered velocity potential in eq. (3) should have been written as

$$
\Phi_{J_{m}, q s t}^{(\mathrm{sc})}=e^{-i \omega t} \sum_{n=|m|}^{\infty} \frac{(n-m) !}{(n+m) !}(2 n+1) i^{(n-m)} h_{n}^{(1)}(k r) A_{n}\left[\Phi_{0} e^{i k_{z} h}+\Phi_{1}(-1)^{n+m} e^{-i k_{z} h}\right] P_{n}^{m}(\cos \theta) P_{n}^{m}(\cos \beta) e^{i m \phi} .
$$

Subsequently, eq. (6) should have been written as

$$
\Phi_{J_{m}, q s t}^{(t)}=e^{-i \omega t} \sum_{n=|m|}^{\infty} \frac{(n-m) !}{(n+m) !}(2 n+1) i^{(n-m)}\left(U_{n}+i V_{n}\right)\left[\Phi_{0} e^{i k_{z} h}+\Phi_{1}(-1)^{n+m} e^{-i k_{z} h}\right] P_{n}^{m}(\cos \theta) P_{n}^{m}(\cos \beta) e^{i m \phi} .
$$

Moreover, the parameter $\Lambda_{n, q s t}^{m}$ given after eq. (13), should have been written as

$$
\Lambda_{n, q s t}^{m}=i^{-m}\left[\Phi_{0} e^{i k_{z} h}+\Phi_{1}(-1)^{n+m} e^{-i k_{z} h}\right] \frac{(n-m) !}{(n+m) !} P_{n}^{m}(\cos \beta) e^{i m \phi} .
$$

In addition, eq. (15) should have been written as

$$
\begin{aligned}
Y_{J_{m}, q s t}= & \frac{8}{(k a)^{2}} \sum_{n=|m|}^{\infty}\left[(-1)^{n+m+1}\left(\frac{\left|\Phi_{1}\right|}{\left|\Phi_{0}\right|}\right)\left[\beta_{n}\left(1+2 \alpha_{n+1}\right)-\beta_{n+1}\left(1+2 \alpha_{n}\right)\right] \sin \left(2 k_{z} h\right)\right. \\
& \left.-\left(\frac{\left|\Phi_{0}\right|^{2}-\left|\Phi_{1}\right|^{2}}{2\left|\Phi_{0}\right|^{2}}\right)\left[\alpha_{n}+\alpha_{n+1}+2\left(\alpha_{n} \alpha_{n+1}+\beta_{n} \beta_{n+1}\right)\right]\right] \frac{(n-m+1) !}{(n+m) !} P_{n}^{m}(\cos \beta) P_{n+1}^{m}(\cos \beta),
\end{aligned}
$$

so that eq. (17) becomes

$$
Y_{J_{m}, s t}=\frac{8}{(k a)^{2}} \sum_{n=|m|}^{\infty} \frac{(n-m+1) !}{(n+m) !}(-1)^{n+m+1}\left[\beta_{n}\left(1+2 \alpha_{n+1}\right)-\beta_{n+1}\left(1+2 \alpha_{n}\right)\right] P_{n}^{m}(\cos \beta) P_{n+1}^{m}(\cos \beta) .
$$

\footnotetext{
a e-mail: mitri@lanl.gov
} 
These changes have been taken into account to reproduce corrected computational plots that should replace those shown in fig. 4(a),(c), fig. 5(a), fig. 6(b),(d), fig. 7(b),(d), and fig. 8(b),(d), respectively. The other published plots are correct and do not require amendments. The corrected figure plots are displayed as follows:
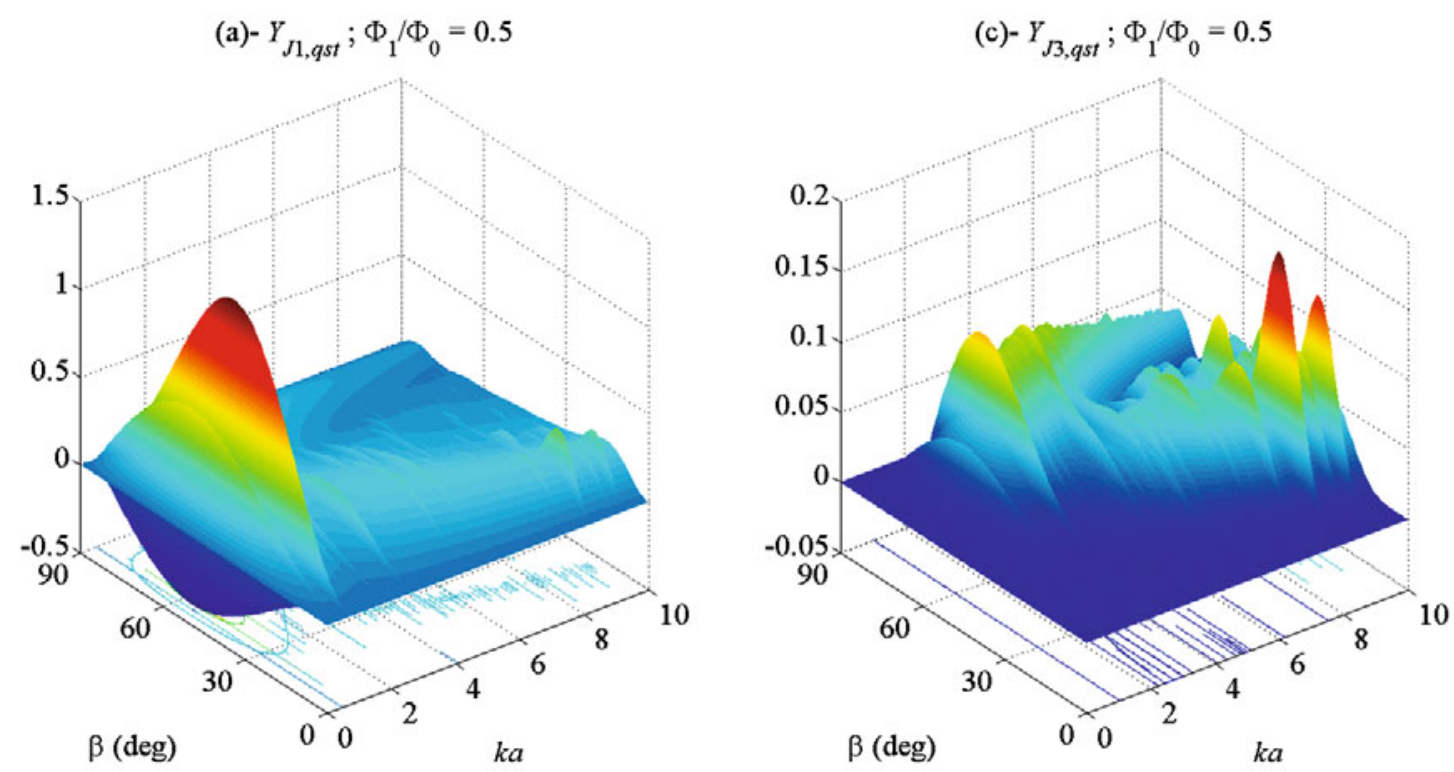

Fig. 4. (Revised - Color online) The plots of the radiation force function where $Y_{J_{m}, q s t}$ are plotted as a function of the dimensionless frequency $k a$ for a spherical air bubble immersed in water. Radiation force function curves are shown for two distinct values of the order of the Bessel vortex beam; (a) $m=1$ (a), and (c) $m=3$. The Bessel beam is composed of quasi-standing waves having $\left|\Phi_{1}\right| /\left|\Phi_{0}\right|=0.5$.

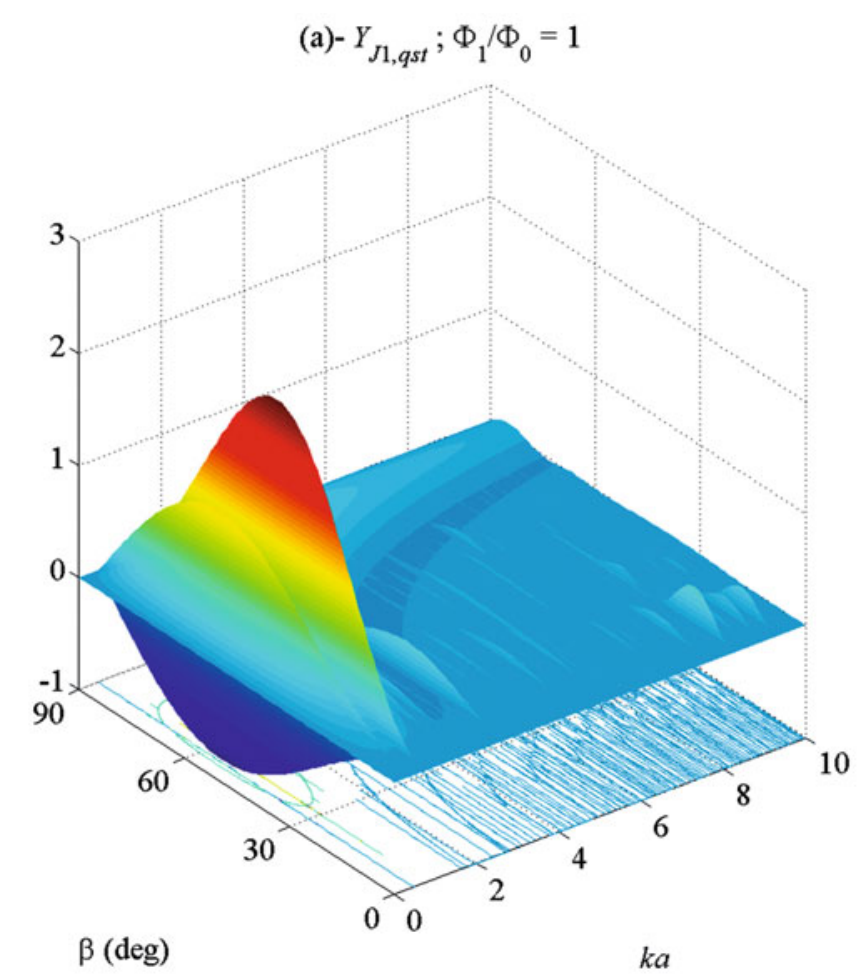

Fig. 5. (Revised - Color online) The plot of the radiation force function where $Y_{J_{m}, q s t}$ for a spherical air bubble immersed in water and placed in a first-order $(m=1)$ Bessel vortex beam composed of equi-amplitude standing waves. 

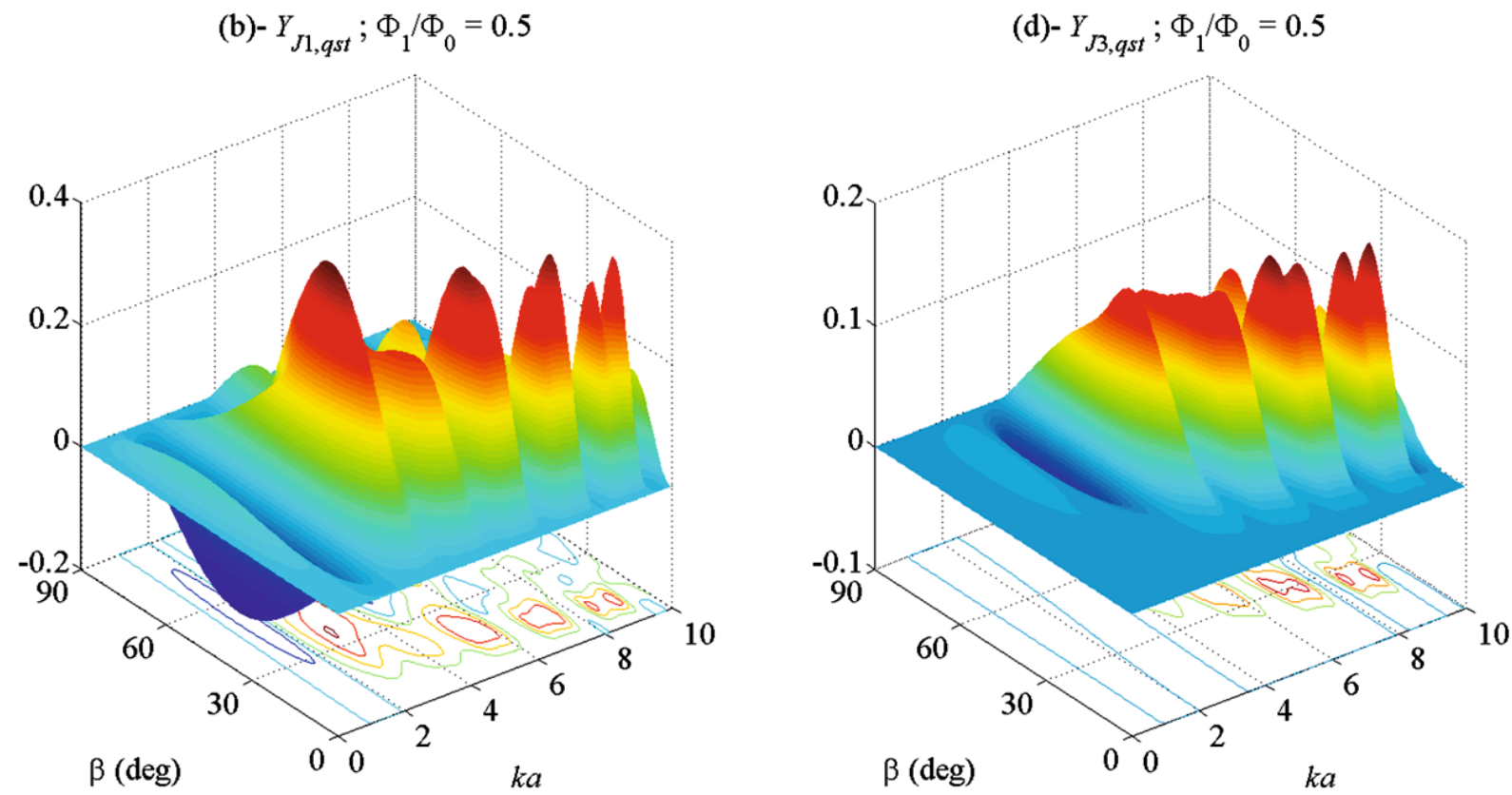

Fig. 6. (Revised - Color online) The plots of the radiation force function where $Y_{J_{m}, q s t}$ for a hexane fluid sphere immersed in water. The first-order (b) and third-order (d) Bessel vortex beams are composed of quasi-standing waves having $\left|\Phi_{1}\right| /\left|\Phi_{0}\right|=0.5$.
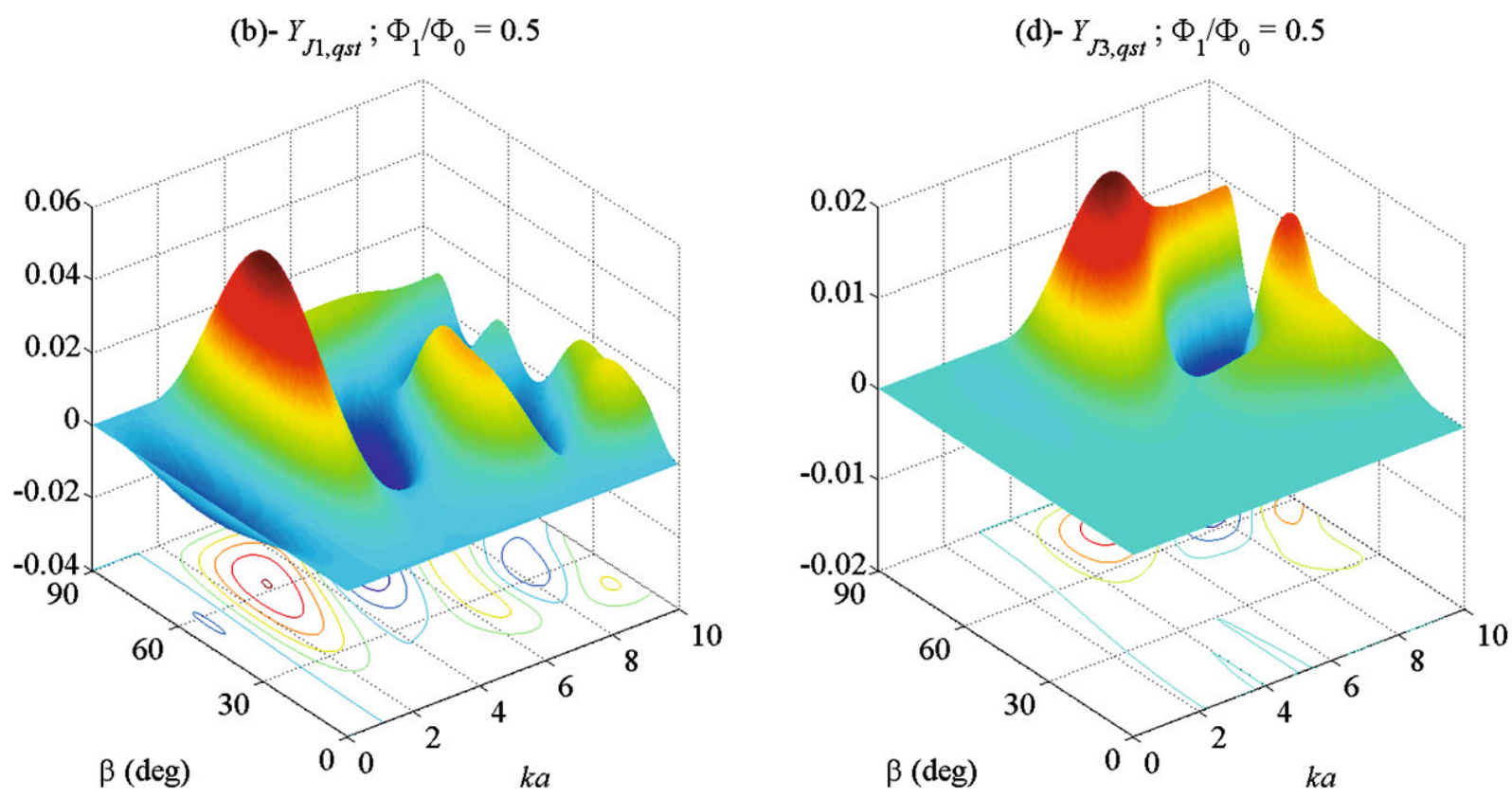

Fig. 7. (Revised - Color online) Same as in fig. 6(b),(d), but the plots correspond to a red blood fluid sphere immersed in water. 

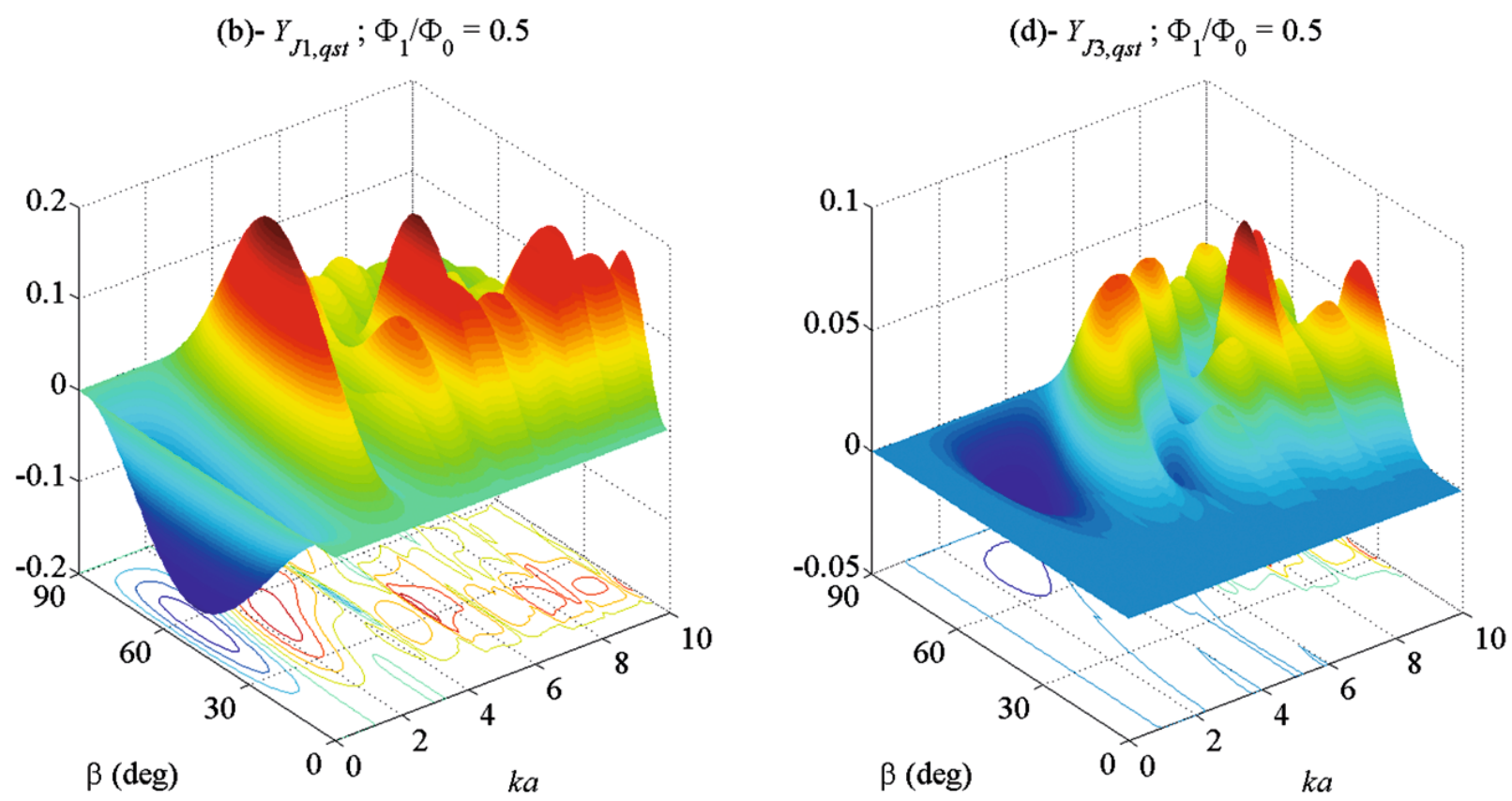

Fig. 8. (Revised - Color online) Same as in fig. 6(b),(d), but the plots correspond to a mercury fluid sphere immersed in water.

\section{References}

1. F.G. Mitri, Eur. Phys. J. E 28, 469 (2009). 